University of Nebraska - Lincoln

DigitalCommons@University of Nebraska - Lincoln

$1-2011$

\title{
Females Are Choosier in the Dark: Environment-Dependent Reliance on Courtship Components and Its Impact on Fitness
}

\author{
Aaron S. Rundus \\ University of Nebraska-Lincoln, arundus2@unl.edu \\ Laura Sullivan-Beckers \\ University of Nebraska-Lincoln, Ibeckers@murraystate.edu \\ Dustin J. Wilgers \\ University of Nebraska-Lincoln, wilgers.spider@gmail.com \\ Eileen Hebets \\ University of Nebraska - Lincoln, ehebets2@unl.edu
}

Follow this and additional works at: https://digitalcommons.unl.edu/bioscihebets

Part of the Behavior and Ethology Commons

Rundus, Aaron S.; Sullivan-Beckers, Laura; Wilgers, Dustin J.; and Hebets, Eileen, "Females Are Choosier in the Dark: Environment-Dependent Reliance on Courtship Components and Its Impact on Fitness" (2011). Eileen Hebets Publications. 38.

https://digitalcommons.unl.edu/bioscihebets/38

This Article is brought to you for free and open access by the Papers in the Biological Sciences at DigitalCommons@University of Nebraska - Lincoln. It has been accepted for inclusion in Eileen Hebets Publications by an authorized administrator of DigitalCommons@University of Nebraska - Lincoln. 
Published in Evolution 65: I (January 20 I I), pp. 268-282; doi: I0.I I I I/j.I558-5646.20I0.0 I I25.x

Copyright (C) 2010 Aaron S. Rundus, Laura Sullivan-Beckers, Dustin J.Wilgers, and Eileen A. Hebets.

Evolution is published by The Society for the Study of Evolution and Wiley-Blackwell. Used by permission.

Submitted April 26, 2010 ; accepted August 10, 2010 ; published online September 2, 2010.

\title{
Females Are Choosier in the Dark: Environment-Dependent Reliance on Courtship Components and Its Impact on Fitness
}

\author{
Aaron S. Rundus, Laura Sullivan-Beckers, Dustin J.Wilgers, and Eileen A. Hebets \\ School of Biological Sciences, University of Nebraska-Lincoln, Lincoln, Nebraska 68588 \\ Corresponding author - A. S. Rundus, email arundus2@unl.edu
}

\begin{abstract}
A broad understanding of multimodal courtship function necessitates knowledge of the potential information content of signal components, the efficacy of signal components in eliciting the appropriate receiver response, and the fitness consequences of mating decisions based upon various signal components. We present data addressing each of these requirements for the multimodal-signaling wolf spider, Schizocosa floridana Bryant. Using diet manipulations, we first demonstrate that both visual and seismic courtship signals are condition-dependent. Next, using high- and low-quantity diet individuals in mate choice trials across manipulated signaling environments, we demonstrate that the seismic signal is crucial for mating success and further show that female choosiness is environment-dependent. Females mated more with high diet males only in the absence of visual signals, showing no discrimination in the presence of visual signals. Finally, by quantifying the number of offspring produced by our mated females, we reveal that a female's mating environment, in conjunction with her potential resource availability, influences her fitness - in environments in which females exerted choice, heavier females produced more offspring. Together, this comprehensive set of experiments demonstrates that female choosiness varies across environments, leading to direct fitness consequences.
\end{abstract}

Keywords: Communication, Schizocosa, signal evolution, spider

Over the past two decades, complex signal function has received increasing attention. Complex signals are defined as having multiple components of either the same (i.e., multicomponent) or different sensory modalities (i.e., multimodal signals) combined into a single display. The complex nature of such animal displays introduces both practical and theoretical challenges to understanding their current function. As such, much work has been devoted to categorizing complex signals, formulating testable hypotheses, and suggesting practical approaches to their study (Partan and Marler 1999, 2005; Candolin 2003; Hebets and Papaj 2005). One such approach relies upon teasing apart components of complex displays and assessing receiver responses to isolated as well as combined components (see Partan and Marler 1999). This is a powerful tool and an essential step toward understanding complex signal function.
Results of studies utilizing this approach provide useful information regarding the necessity/sufficiency of various signal components as well as information on interactions between components/signals (see Partan and Marler 1999). However, such studies provide no direct information on potential signal content. Ultimately, signal form, regardless of its level of complexity, is thought to result from both content-based (strategic design) and efficacy-based (tactical design) selection pressures (Guilford and Dawkins 1991) and as such, both need to be simultaneously considered to fully understand complex signal function.

Content-based selection refers to selection on the information encoded in the signal (e.g., species identity, body condition, location, etc.). Examples of content-based hypotheses of complex signal function include "multiple messages," where different signal components encode 
different information, and "redundant signals," where different signal components encode the same information, potentially enabling a perceiver to increase the accuracy of their response (Moller and Pomiankowski 1993; Johnstone 1996). In contrast, efficacy-based selection relates to selection for signal transmission and/or reception and processing (Guilford and Dawkins 1991; Endler 1993; Owings and Morton 1998; for empirical example see Fleishman 1986). For example, efficacy-based hypotheses propose that complex signals aid in overcoming variability in the signaling environment and/or the receiver's sensory or processing systems (Candolin 2003; Hebets and Papaj 2005). Ultimately, successful communication involves the effective production, transmission, reception and processing of signals, with selection likely acting on all steps in the process.

In addition to the simultaneous exploration of both content- and efficacy-based selection pressures, studying complex signal function requires an acknowledgment of the plasticity often present in the receivers, or targets, of complex signals. Receivers may vary in their response to signals and/or components based upon their internal state (e.g., hunger level, age, reproductive physiology, etc.) or on external factors (e.g., presence of predators, social environment, etc.). For example, a female's choice of a mate has been shown to depend on her age (Moore and Moore 2001; Coleman et al. 2004), her condition (Hebets et al. 2008; Eraly et al. 2009; reviewed in Jennions and Petrie 1997), and even her experience (Collins 1995; Wagner et al. 2001; Hebets 2003; Hebets and Vink 2007). Given the rapidly accumulating evidence of variability in female mate choice and/or preference based upon internal factors such as those mentioned above, it is not surprising that females also alter their choice based on external factors, such as current environmental conditions (both biotic and abiotic). For example, a female's preference for conspicuous males may be reduced or reversed when predation risk is high (e.g., Forsgren 1992; Hedrick and Dill 1993; Godin and Briggs 1996; Gong and Gibson 1996; Johnson and Basolo 2003). Recent evidence from sticklebacks even suggests that females alter their reliance on signals in different sensory modalities under variable environmental conditions. Specifically, stickleback females relied more on visual cues than olfactory cues in clear water, whereas they relied more on olfactory cues than visual cues in turbid water (Heuschele et al. 2009). Fish populations living permanently under environmental conditions that favor one over the other sensory channel may even evolve genetic population differences in female mate choice behavior (e.g., Tobler et al. 2008). The recent increase in examples of variable female preferences highlights the notion that an understanding of the variability present among, and even within, receivers is crucial for our understanding of signal evolution. Although many of the proposed efficacy-based hypotheses of complex signal function pertain to receiver variability (Hebets and Papaj 2005), surprisingly few of these hypotheses have been empirically tested.
We suggest that a major step toward understanding complex signal function requires knowledge of three key components. (1) The potential information content of signal components, (2) the efficacy of complex signal components with regard to their ability to elicit a desired response, and (3) the fitness consequences of mating decisions based upon various signal components. Additionally, when exploring both the efficacy of complex signal components and the fitness consequences of mating decisions based upon these components, receiver variability needs to be taken into consideration. Here, using an experimental design that manipulates both signaling environment and foraging history (i.e., adult body condition), we examine all three of these components in the wolf spider Schizocosa floridana Bryant, with a focus throughout on receiver variability.

\section{General Methods}

Schizocosa floridana is found in the southeastern United States typically in oak leaf litter (Dondale and Redner 1978; G.B. Edwards and A.S. Rundus, pers. obs.). Although their courtship display has not been formally described, Stratton (2005) categorizes their courtship as multimodal-consisting of a seismic component (formally described below) and a visual component involving a foreleg tap. Mature males possess dark pigmentation, which is fixed at maturation, on their foreleg patella, tibiae, and metatarsus (Figure $1 \mathrm{~A}_{1}$ ), but do not possess brushes as seen in some other members of the genus (Stratton 2005). In addition to visual and seismic signaling, chemical-based signaling is not uncommon in arachnids including members of the genus Schizocosa (Ayyagari and Tietjen 1986; Gaskett 2007). However, there is no evidence to suggest that a chemical component of male courtship influences a female spider's decision to copulate and we therefore focused only on the visual and seismic components of male courtship.

Immature male and female $(N=404) S$. floridana were collected from the field on January 16-18, 2008, in Alachua County, FL. Spiders were subsequently brought to the laboratory where they were weighed (Ohaus Adventurer Pro AV64 Pine Brook, NJ) and housed individually in $5.9 \mathrm{~cm} \times 5.9 \mathrm{~cm} \times 7.7 \mathrm{~cm}$ clear plastic containers (Amac Plastic Products, Petaluma, CA) with visual barriers between containers. They were maintained on a 12L:12D light cycle and provided with a constant source of water via soaked cotton dental rolls (Dynarex Corporation, Orangeburg, NY).

To generate variation in the physical condition of both male and female spiders, all individuals were randomly assigned to one of two diet treatments upon collection: (1) Low-quantity diet (LD)-one cricket (Acheta domesticus) (Bassetts cricket ranch, CA) visually size-matched to the body length of the spider (prosoma + opisthosoma) once 

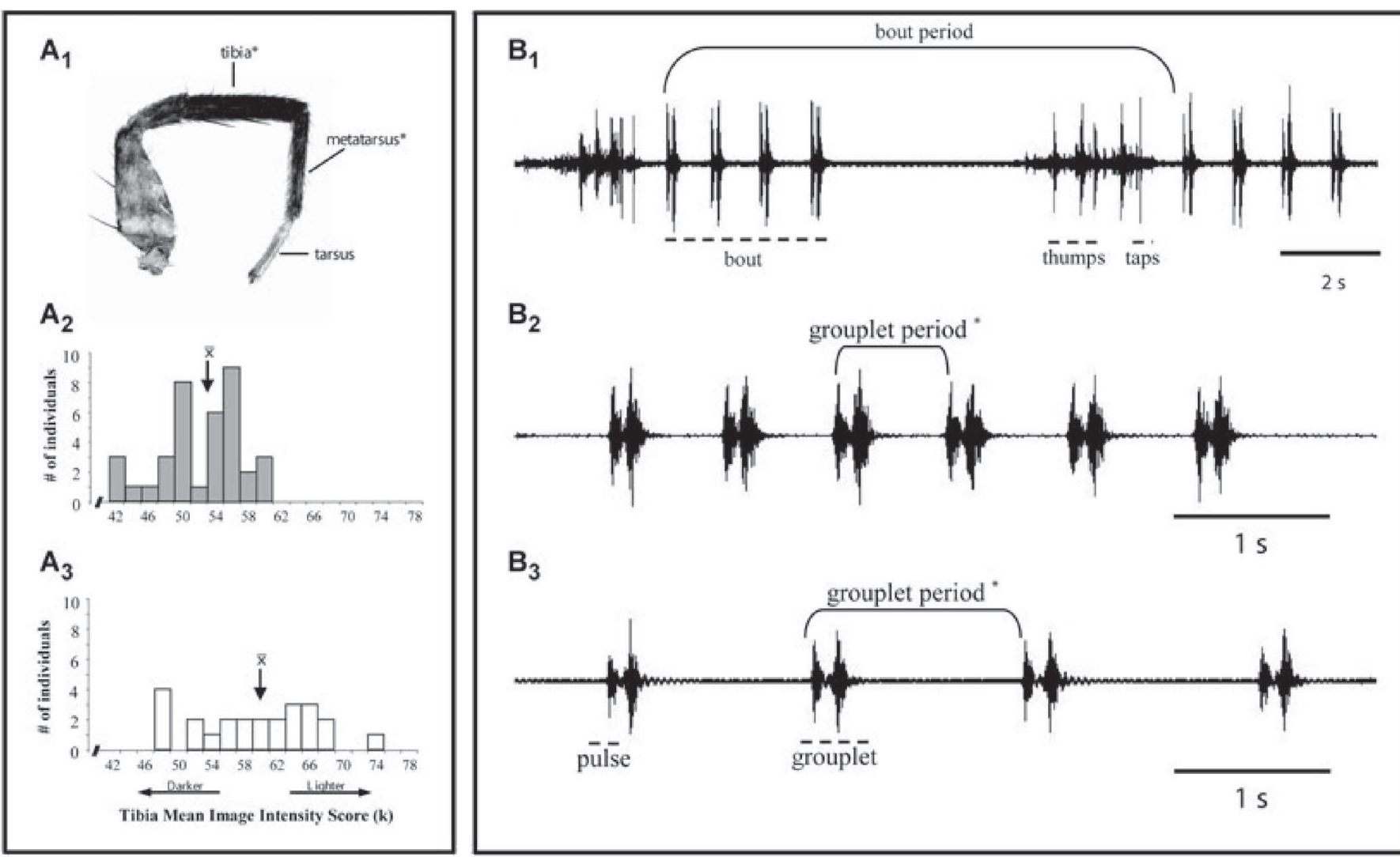

Figure I. (A) Male foreleg of S. floridana. Panel $A_{1}$ indicates the pigmented (tibia and metatarsus) and control (tarsus) segments measured in this study. Panels $A_{2}$ and $A_{3}$ show distributions of male mean tibia darkness of high-diet and low-diet males, respectively. Lower image intensity scores indicate darker segments. (B) Waveforms of a typical male signal. Panel $B_{1}$ shows two signaling bouts, including the stridulatory thumps, percussive leg taps, and tremulation pulses. Panels $B_{2}$ and $B_{3}$ are a more fine-scale depiction of one tremulation bout, composed of pulses grouped into doublets. Panel $B_{2}$ is a single bout of a high-diet male. Panel $B_{3}$ is a single bout from a low-diet male. Note that panels $B_{2}$ and $B_{3}$ are on the same time scale. Grouplet period differs significantly between high and low diet males (see text).

every two weeks, or (2) High-quantity diet (HD) - one cricket size-matched as above twice per week. Spiders were examined every two days for molts to determine the date of maturation. In females, diet was manipulated to examine the relationship between diet and mate choice, as a relationship has been previously found in another Schizocosa wolf spider (Hebets et al. 2008). To calculate a body condition score, body size measures were taken on spiders (preserved in $70 \%$ ethanol) at the conclusion of all experiments. Body size could not be measured on some individuals due to decomposition or postcopulatory cannibalism, which also excluded them from body condition estimates (see below).

\section{Experiment I-Quantifying Ornamentation and Seismic Signals}

To gather data on the potential information content of both visual and seismic male courtship signals, we quantified foreleg ornamentation and seismic signals for HD and LD males upon maturation.

\section{Experiment 1: Methods}

\section{Quantifying foreleg ornamentation}

To examine the influence of diet on male foreleg coloration (a presumed secondary sexual ornament, see Stratton 2005), a subset of males ( $N=61$; HD: $N=$ 37, LD: $N=24$ ) were sacrificed and frozen at $-4^{\circ} \mathrm{C}$ for just over 1 year ( 400 days). Each male's right foreleg was removed and allowed to thaw. Thawed legs were placed on a clear microscope stage and covered with a glass slide to flatten all segments to a single plane. Legs were manually positioned to minimize glare. The lateral side of each foreleg was digitally photographed using a stereoscope (Leica MZ16, Bannockburn, IL) and a Spot Flex digital camera (Model 15.2 64 MP, Diagnostic Instruments, Inc. Sterling Heights, MI), under a 3.2 $\times$ objective and $1.2 \times$ camera coupler. Legs were illuminated from above by 150 watt Lumina dual fiber optic lights (Chiu Technical Corporation, Kings Park, NY). All leg images were captured on the same day using the same settings and light levels, enabling direct comparisons among individuals. 
Foreleg coloration for $S$. floridana males was quantified following the methods outlined by Shamble et al. (2009). Briefly, digital images were imported into Adobe Photoshop CS2 where we analyzed the tibia, metatarsus, and tarsus segments for the following two measures: (1) segment area (no. of pixels) and (2) mean segment image intensity (i.e., darkness, a numerical reading where 255 is white and 0 is black). Each of these measurements was taken once. The percent of each segment covered by dark coloration was also calculated using the threshold function following Shamble et al. (2009). The threshold image intensity value was calculated as 1 standard deviation darker than the population mean, measured as the image intensity across the pigmented tibia and metatarsus.

Based on predictions of handicap models of sexual selection, sexually selected ornaments are expected to express greater condition dependence than nonsexually selected traits (Cotton et al. 2004). Therefore, we compared the ornamented leg segments (the sexually dimorphic tibia and metatarsus) to a nonornamented leg segment (the monomorphic tarsus). Using a repeated-measures multivariate analysis of variance (MANOVA), we analyzed the difference in mean image intensity for each of these segments within an individual, across the diet treatments. A significant interaction between diet treatment and segment, within an individual, would indicate the darkness of the segments is changing at different rates for high- and lowdiet treatment individuals. All nonnormally distributed data were $\log$ transformed. If transformation did not result in normal distribution nonparametric tests were used. All analyses were performed in JMP version 6 (SAS Institute Inc., Cary, NC). Means and their associated standard errors are presented throughout.

\section{Quantifying the seismic signal}

We used a subset of males (15 HD and 15 LD) for seismic signal analysis that were not included in the ornamentation analysis. Male age ranged from 36 to 60 days post maturation. The average age of HD males was 54.6 and the average age of LD males was 49.12 days.

All recordings were made on a vibration isolation table (Minus K 50BM-8C, Minus K Technology, Inglewood, CA) in a soundproofed enclosure $(50 \mathrm{~cm} \times 37 \mathrm{~cm} \times 43 \mathrm{~cm}$ lined with loaded vinyl PSA and soundproof foam, Super Soundproofing Co., San Marcos, CA) illuminated with a Vita-Lite full spectrum florescent bulb (Duro-Test Lighting Inc., Philadelphia, PA). Recordings were made using a laser vibrometer (Polytec PDV100), set for a peak velocity measurement range of $\pm 20 \mathrm{~mm} / \mathrm{s}$, with a low pass filter at $22 \mathrm{kHz}$, and at a 24 bit $48 \mathrm{kSa} / \mathrm{s}$ sample rate. Digital output from the vibrometer was recorded on an Apple PowerBook using a power 1401 A-to-D converter and Spike 2 version 5 (Cambridge Electronic Design Ltd, Cambridge, UK). All vibrational recordings were also filmed using a Logitech Webcam Pro 9000 (Logitech, Fremont, CA), mounted on the inside top of the soundproof chamber. Video from each vibrational recording was synchronized with the vibrometer output using Spike 2. All recordings were exported as uncompressed WAV files for signal analysis.

Prior to each recording, one randomly chosen virgin mature female spider, 22 days post maturation, was allowed to deposit silk onto a piece of Whatman no. 1185 $\mathrm{mm}$ filter paper for a period of $12 \mathrm{~h}$. A different female was used for every male. During recording trials, the impregnated filter paper was suspended on a flat, circular stainless steel ring $(18.5 \mathrm{~cm}$ diameter and $\mathrm{cm}$ wide) raised $2 \mathrm{~cm}$ above the bottom of the enclosure. A $0.5 \mathrm{~cm} \times 0.5 \mathrm{~cm}$ piece of retroreflective tape (3 M Diamond Grade, $3 \mathrm{M}$, Saint Paul, MN) was placed in the center of the filter paper to increase the signal strength of the vibrometer. A transparent plastic acetate wall was placed around the perimeter of the metal ring, but not in contact with the filter paper, to prevent the spider from escaping. During each recording trial, a single male was placed on the filter paper and allowed to court for a period of $5 \mathrm{~min}$. We predicted that differences in signaler condition would be more evident after $4 \mathrm{~min}$ of courtship if signaling was energetically costly. For this reason, all signals within the fifth minute of each recording were analyzed for all males. All signal measurements were made in Raven Pro (version 1.3, Cornell Laboratory of Ornithology, Ithaca, NY).

To test for differences among HD and LD males, we used a nominal logistic regression, with male diet as the response variable and standardized signal traits as predictor variables. In essence, the nominal logistic regression tests the direct influence of a signal trait at predicting which diet treatment a male was in by holding all other signal variables constant.

\section{Experiment 1: Results}

At the time of collection from the field, there was no difference in initial weights for individuals of either sex across diet treatments (females, HD: $N=60, \bar{x}=16.3 \pm 0.4 \mathrm{mg}$; LD: $N=142, \bar{x}=15.6 \pm 0.3 \mathrm{mg}$; Wilcoxon test, $Z=1.65, P=0.1$ and males, HD: $N=101, \bar{x}=13.8 \pm 0.3 \mathrm{mg}$; LD: $N=101, \bar{x}$ $=14.0 \pm 0.3 \mathrm{mg}$; $t$-test, $t_{200}=0.52, P=0.61$. On average, individuals were on their respective diet manipulations for $26 \pm 0.4$ days before they matured, and $48 \pm 0.4$ days before the mate choice trial. When weighed on the date of mate choice trials, HD individuals had gained significantly more mass than LD individuals (Weight gain: females, HD: $N=$ $60, \bar{x}=43 \pm 0.7 \mathrm{mg}$; LD: $N=142, \bar{x}=12 \pm 0.4 \mathrm{mg}$; Wilcoxon test, $Z=11.22, P<0.001$ and males, HD: $N=101, \bar{x}$ $=11 \pm 0.3 \mathrm{mg} ; \mathrm{LD}: N=99, \bar{x}=5 \pm 0.3 \mathrm{mg}$; Wilcoxon test, $Z$ $=10.34, P<0.001)$. Additionally, body size (cephalothorax width), a trait that is fixed at maturation, was significantly larger for HD individuals than LD individuals (females, HD: $N=45, \bar{x}=2.30 \pm 0.02 \mathrm{~mm}$; LD: $N=67, \bar{x}=2.06 \pm 0.02$ $\mathrm{mm}$; $t$-test, $t_{110}=8.17, P<0.001$ and males, HD: $N=99, \bar{x}=$ $2.18 \pm 0.01 \mathrm{~mm}$; LD: $N=94, \bar{x}=2.00 \pm 0.01 \mathrm{~mm}$; Wilcoxon test, $Z=7.30, P<0.001)$. 


\section{Foreleg ornamentation}

HD males had significantly larger (i.e., area in $\mathrm{mm}^{2}$ as estimated by pixel number) foreleg segments than LD males (Table 1). All foreleg segments were generally darker (e.g., for tibia differences see Figure $1 \mathrm{~A}_{2}$ and Figure $1 \mathrm{~A}_{3}$ ) and were covered with more dark coloration in HD versus LD males (Table 1). The ornamented segments (tibia and metatarsus) were also significantly darker than the nonornamented segment (tarsus). The difference in mean segment darkness (log transformed) between the ornamented and nonornamented segments was also significantly greater for HD versus LD males (repeated measures MANOVA, diet: $F_{1,59}=31.5, P<0.001$; segment: $F_{2,58}$ $=2853.5, P<0.001$; diet $\times$ segment: $F_{2,58}=10.1, P<0.001$; Table 1). Additionally, we found male foreleg coloration to be dependent upon male condition, with better condition males expressing darker forelegs. This was indicated by significant negative correlations (darker legs have lower image intensity scores) between image intensity scores (all log transformed) and our body condition index (mass (g)/ cephalothorax width $(\mathrm{mm}))$ for the tibia $\left(N=44, R^{2}=0.23\right.$, $\left.F_{1,42}=12.34, P=0.001\right)$ and metatarsus $\left(N=44, R^{2}=0.15\right.$, $\left.F_{1,42}=7.57, P=0.009\right)$, but not the nonornamented tarsus segment $\left(N=44, R^{2}=0.07, F_{1,42}=3.17, P=0.08\right)$.

\section{Seismic signaling}

Schizocosa floridana males have a complex seismic courtship display (Figure $1 \mathrm{~B}_{1}$ ) that incorporates three vibration-production mechanisms-stridulation, percussion, and tremulation. Males produce a low-frequency stridulatory pulse, referred to here as a "thump" - produced strictly via stridulation with no percussive component. Males also strike their first pair of legs on the substrate in a quick succession, which we refer to as "leg taps." In a typical bout of courtship, males produce the thump at regular intervals until increasing the rate of thumps just before adding three to four leg taps, and finally starting the tremulation portion of the display. The tremulation produces "pulses" of vibration in discrete groups. Based on visual inspection (the abdomen conspicuously bobs with each pulse), a single pulse represents a discrete muscle contraction. Pulses normally occur in pairs or triplets, which we referred to as a "grouplet." Males produce four to six grouplets in sequence, which we refer to as a signal bout. Signal bouts are separated by at least $2 \mathrm{sec}$ with no tremulation.

To quantify the tremulation portion of the display, we quantified the following traits: bout period (the time between the initiation of successive bouts); total signaling time (the amount of time signaling in the fifth minute); grouplet period (the time between the initiation of each grouplet); pulse rate (the average number of pulses/grouplet duration); average number of pulses/bout; and pulse frequency (the average frequency of the first pulse of each grouplet in the first bout). See Figure 1B for further clarification. The tremulation signal has most of its energy in a narrow frequency range, enabling us to measure signal frequency from a waveform by counting the number of cycles per unit time. We also include measures of the number of thumps and leg taps in our analysis.

HD males spent more time signaling in the fifth minute and had shorter grouplet periods (Table 2; Figures $1 \mathrm{~B}_{2}$ and $B_{3}$ ). The grouplet period, although faster in HD males, did not alter the amount of silent time between signaling bouts. In other words, HD males produced grouplets more quickly within a bout, but bout period did not differ between HD and LD males. We also found no differences in frequency, pulse period, pulses/bout, total thumps, or total leg taps between HD and LD males (Table 2).

Table I. Effects of diet quantity manipulation on male foreleg characteristics.

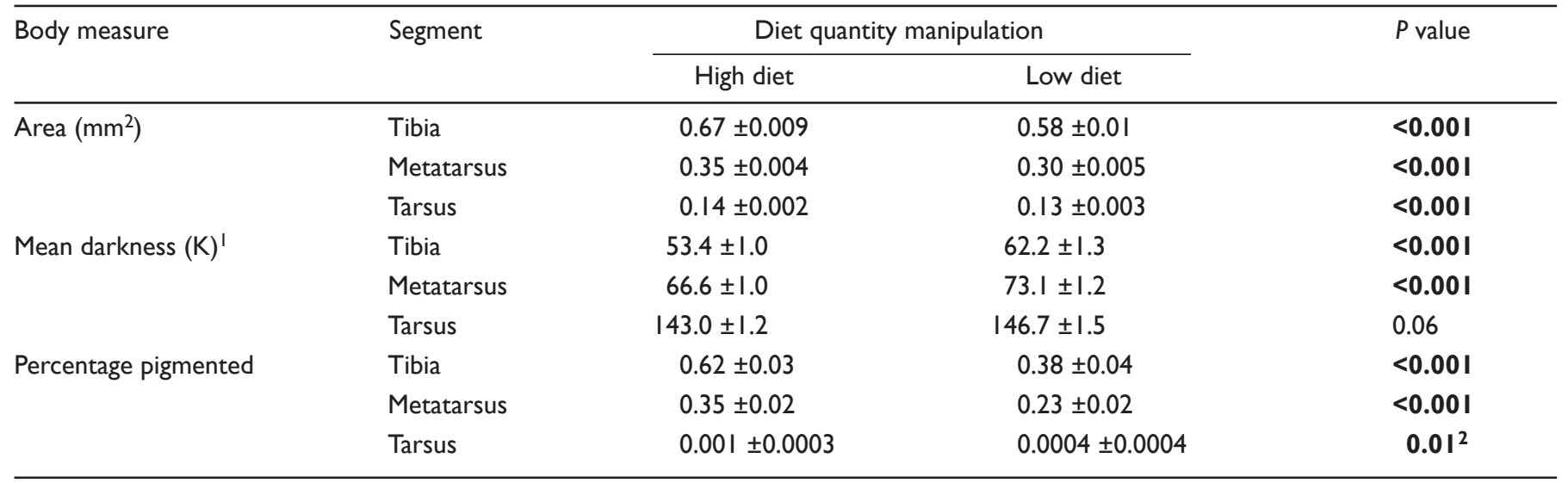

I. Darkness quantified as mean image intensity, where values of $\mathrm{K}$ range from 0 (black) to 255 (white). $P$-values reported from a $t$-test on log transformed data.

2. $P$ value reported from nonparametric two-sample Wilcoxon tests. 


\section{Experiment 2-Efficacy of Male Courtship Components}

One way to determine signal efficacy is to evaluate a receiver's response to a signal in isolation. If a receiver responds appropriately, a signal can be assumed to be effective. Although a lack of a response does not necessarily imply an ineffective signal, assessing receiver responses to isolated signals is a good first approach to determining signal efficacy. Thus, to determine the efficacy of $S$. floridana visual and seismic courtship signals, we manipulated the signaling environment to prevent the transmission of each signaling modality independently, and subsequently allowed virgin females and males to interact. This also enabled us to determine whether signals were necessary or sufficient for copulation.

\section{Experiment 2: Methods}

A total of 108 females $(\mathrm{HD}=60 ; \mathrm{LD}=48)$ and 108 males $(\mathrm{HD}=55 ; \mathrm{LD}=53)$ from our initial collection were used in single mate choice trials ( 1 male and 1 female). Trials were run in a fully crossed $2 \times 2$ experimental design with a visual treatment of light versus dark (visual present $(\mathrm{V}+) / \mathrm{ab}-$ sent $(\mathrm{V}-)$, respectively) and a seismic treatment of filter paper substratum versus granite substratum (seismic present (S+)/absent (S-), respectively). Granite, like other types of rock, does not propagate seismic signals effectively (Elias et al. 2004). The experimental protocol in this study was similar to Hebets (2005) and identical to Rundus et al. (2010).

Visual present trials $(\mathrm{V}+)$ were illuminated using two Vita-Lite full spectrum 30-watt florescent bulbs (Duro-Test Lighting Inc.) whereas visual absent trials $(\mathrm{V}-)$ were conducted in complete darkness with behavioral observations facilitated by the use of Rigel 3200 night vision goggles (Rigel Optics Inc., Washougal, WA) and a Supercircuits IR20 infrared illuminator (Supercircuits, Austin, TX). The IR illuminator emitted wavelengths of $\sim 850 \mathrm{~nm}$ and data on both wolf spiders, and on the wandering spider Cupiennius salei, provide no indication that they can detect IR wavelengths in excess of $800 \mathrm{~nm}$ (DeVoe et al. 1969; DeVoe 1972; Barth 2002).
Preliminary mate-choice trials were conducted to determine whether our testing apparatus provided a suitable environment for courtship and mating. Due to low copulation success, arenas were modified in the following manner: a visual barrier (image of oak leaves photographed at a Schizocosa collection site) was wrapped around the outside the arena wall and small brown construction paper cutouts leaves were glued on the rim of the wall, providing shade/contrast from the overhead light source. This modified arena design resulted in increased courtship and mating, and was subsequently used in all experimental trials.

Groups of four V+ trials were always run back-to-back with groups of four $\mathrm{V}$ - trials with the initial treatment randomly chosen. $\mathrm{V}+$ trials were comprised of two replicates each of $\mathrm{V}+/ \mathrm{S}+(N=27)$ and $\mathrm{V}+/ \mathrm{S}-(N=26)$. Similarly, $\mathrm{V}-$ trials consisted of two replicates each of $\mathrm{V}-/ \mathrm{S}+(N=31)$ and $V-/ S-(N=24)$. Thus, at any one time, four pairs of females and males were observed simultaneously (e.g., $2 \times$ $\left.\mathrm{V}+/ \mathrm{S}+, 2 \times \mathrm{V}+/ \mathrm{S}^{-}\right)$. Mate choice trials in the seismic-present treatment $(\mathrm{S}+)$ were run in circular plastic arenas measuring $12.5 \mathrm{~cm}$ diameter (Pioneer Plastics, Inc. Dixon, KY) lined on the bottom with a piece of Whatman no. 1 Filter paper (Schleicher and Schuell, Keene, NH). Seismic signal transmission was prevented in the seismic-absent treatment (S-) by running trials in bottomless arenas of the same size placed on a piece of granite.

Approximately $24 \mathrm{~h}$ prior to testing, females were provisioned with a single cricket, size-matched to one half of the female's body size in an effort to minimize the likelihood of precopulatory cannibalism. On their testing day, immediately prior to trials, males and females were weighed (Ohaus Adventurer). HD or LD females were then each placed in their assigned testing arenas. After a 5-min acclimation period, either an HD or LD male was placed in each testing arena under an inverted glass vial. Trials commenced with the removal of the inverted vials and each pair was allowed to interact for up to $30 \mathrm{~min}$.

All trials were observed and scored for the following behaviors: copulation, cannibalism, and attempted mounts of the female. Instantaneous sampling of all male behavior including courtship was also conducted at 5-min intervals, giving a total of six samples per trial. The latency to

Table 2. Signal variation of males on high diet (HD) and low diet (LD) treatments. Means \pm standard errors shown. Chi-square values derived from logistic regression that included all signal traits.

\begin{tabular}{lrccc}
\hline Signal parameter & \multicolumn{1}{c}{ High diet } & Low diet & Chi square & $P$ value \\
\hline Total time signaling & $16.59 \pm 1.93$ & $14.03 \pm 1.11$ & 5.1048 & $\mathbf{0 . 0 2 4}$ \\
Bout period & $10.58 \pm 0.94$ & $12.32 \pm 1.46$ & 1.372 & 0.242 \\
Grouplet period & $0.78 \pm 0.04$ & $0.94 \pm 0.05$ & 4.669 & 0.030 \\
Frequency & $318.21 \pm 9.10$ & $298.89 \pm 11.41$ & 0.2868 & 0.592 \\
Pulse period & $0.14 \pm 0.01$ & $0.14 \pm 0.01$ & 0.1951 & 0.659 \\
Pulses/bout & $11.07 \pm 1.41$ & $12.8 \pm 2.13$ & 0.0288 & 0.865 \\
Total thumps & $15.47 \pm 2.25$ & $10.87 \pm 2.29$ & 0.0674 & 0.795 \\
Total leg taps & $8.00 \pm 1.39$ & $8.67 \pm 1.86$ & 2.925 & 0.087 \\
\hline
\end{tabular}


Table 3. Table of effects for nominal logistic regression models (A) in experiment 2 with visual treatment condition, female and males diet conditions, and female age as predictors and copulation success as the response variable, and (B) in experiment 3 with male masking treatment, male diet treatment, visual treatment condition, and male and female age as predictor variables with copulation success as the response variable.

\begin{tabular}{|c|c|c|c|}
\hline Source & $\mathrm{df}$ & Chi Square & $P$ value \\
\hline \multicolumn{4}{|l|}{ (A) } \\
\hline F Diet & 1 & 0.341 & 0.599 \\
\hline M Diet & 1 & 0.162 & 0.687 \\
\hline Visual & 1 & 7.909 & 0.005 \\
\hline F Diet $\times$ M Diet & 1 & 1.329 & 0.249 \\
\hline F Diet $\times$ Visual & 1 & 3.643 & 0.056 \\
\hline M Diet $\times$ Visual & 1 & 5.646 & 0.018 \\
\hline F Diet $\times$ M Diet $\times$ Visual & 1 & 3.511 & 0.061 \\
\hline FAge & 1 & 0.212 & 0.646 \\
\hline \multicolumn{4}{|l|}{ (B) } \\
\hline Masking & 1 & 3.277 & 0.070 \\
\hline Diet & 1 & 0.005 & 0.942 \\
\hline Visual & 1 & 4.723 & 0.029 \\
\hline Masking $\times$ Diet & 1 & 0.141 & 0.707 \\
\hline Masking $\times$ Visual & 1 & 0.162 & 0.687 \\
\hline Diet $\times$ Visual & 1 & 5.480 & 0.019 \\
\hline Masking $\times$ Diet $\times$ Visual & 1 & 0.012 & 0.912 \\
\hline FAge & 1 & 0.258 & 0.612 \\
\hline MAge & 1 & I.345 & 0.246 \\
\hline
\end{tabular}

first courtship and copulation was also recorded. All statistical tests ( $t$-test, chi square test, and nominal logistic regression, Wilcoxon test) were carried out using JMP version 7.01 (SAS Institute Inc.).

\section{Experiment 2: Results}

At the time of testing, females and males on the HD treatment had a significantly higher body condition score than those on the LD treatment (Females: LD $\bar{x}=0.013 \pm$ $0.0006 \mathrm{~g} / \mathrm{mm}, \mathrm{HD} \bar{x}=0.026 \pm 0.0005 \mathrm{~g} / \mathrm{mm}$; $t$-test, $t_{76}=$ 18.302, P < 0.0001; Males: LD $\bar{x}=0.009 \pm 0.0002 \mathrm{~g} / \mathrm{mm}$, HD $\bar{x}=0.011 \pm 0.0002 \mathrm{~g} / \mathrm{mm}$; $t$-test, $\left.t_{98}=8.33, \mathrm{P}<0.0001\right) . \mathrm{A}$ total of 34 copulations took place with only one occurring in a seismic absent treatment $(\mathrm{V}-/ \mathrm{S}-)$, indicating that the seismic signal is crucial for mating success. To explore the relationships between signaling environment, female and male diet treatment, and female age (previously shown to be important in female choice in S. ocreata; Uetz and Norton 2007), we ran a nominal logistic regression using all $\mathrm{S}+$ trials with these variables as predictors and the occurrence of copulations as the response variable. The overall model was significant $\left(\bar{x}=17.20, R^{2}=0.217, P=0.028\right)$. Neither diet treatment, regardless of sex, nor female age influenced copulation success (Table 3 ). The visual treatment, however, did predict copulation success, with males being more likely to copulate in the visual present $(\mathrm{V}+)$ condition (Figure 2A; Table 3). Furthermore, we found a significant interaction between the visual treatment and male diet (Figure 2A; Table 3), with HD males being more likely to copulate than LD males in the visual absent condition $(\mathrm{V}-)$, and no significant difference in likelihood to copulate between the two diet treatments in the visual present condition $(\mathrm{V}+)$.

Age at the time of testing ranged from 15 to 27 days post maturation for females $(\bar{x}=21.2)$ and $15-37$ days for males $(\bar{x}=20.4)$. Due to the later maturation of low diet individuals, HD females and males were significantly older than LD females and males at the time of testing (Table S1A 1 ). However, we found no significant age difference in females or males in trials in which a mating occurred compared to trials with no mating (Table $\mathrm{S}_{1} \mathrm{~A}_{2}$ ). Furthermore, there was no significant difference between the age of females that mated with either HD or LD males (LD males: $N=14, \bar{x}=$ $21.14 \pm 0.96$ days post maturation; HD males: $N=20, \bar{x}=$ $21.1 \pm 0.85$ days post maturation; Wilcoxon test: $Z=0.212$, $P=0.832)$.

Male diet did not appear to influence motivation to court (Table S2A $\mathrm{A}_{1}$ ) as there was no difference between HD and LD males with regard to the latency from the start of the trial to their first bout of courtship, the latency from the start of the trial to copulation, or the latency from the first bout of courtship to copulation. To obtain snapshots into male courtship effort, we conducted instantaneous sampling every $5 \mathrm{~min}$ (a total of six samples per trial), which also revealed no significant difference in the amount of courtship between HD and LD males. Similarly, the amount of courtship did not differ significantly between the four environmental treatments, however, we did find differences in the latency to the first bout of courtship across treatments with a longer latency in the $\mathrm{V}+/ \mathrm{S}-$ condition compared to the other three treatments (Table S2 $\mathrm{A}_{2}$ ).

Males who courted more, as measured by instantaneous samples every $5 \mathrm{~min}$ of the trial, were more likely to copulate (copulation: $\bar{x}=0.86 \pm 0.05$ samples; no copulation: $\bar{x}$ $=0.61 \pm 0.04$ samples; Wilcoxon test: $Z=3.22, P=0.0013$ ). However, as previously illustrated, the amount of courtship did not differ between male diet treatment or signaling environment. Of the males who copulated, the latency to copulation differed among the environmental treatment conditions with a shorter latency in the $\mathrm{V}+$ conditions than in the $\mathrm{V}-$ conditions (Figure $2 \mathrm{~B}$ ).

\section{Experiment 3: Reversible Male Visual Masking}

Results from the signal efficacy experiment (Experiment 2) indicated that HD males had a mating advantage in the dark $(\mathrm{V}-/ \mathrm{S}+)$ as compared to LD males, but 

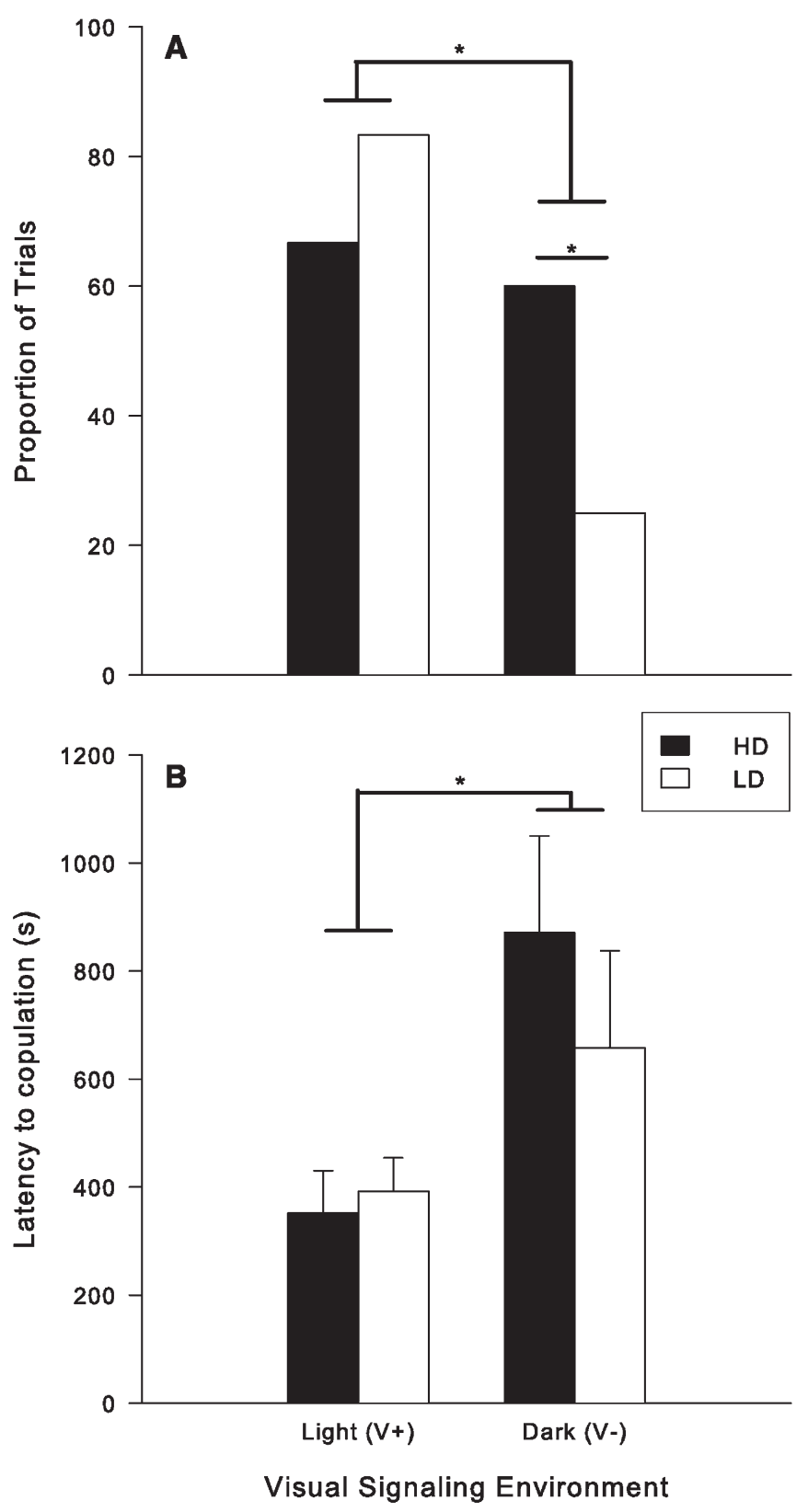

Figure 2. (A) Panel shows the proportion of mate choice trials in which HD and LD males copulated in the light $(\mathrm{V}+/ \mathrm{S}+)$ and dark $(\mathrm{V}-/ \mathrm{S}+)$ testing conditions. Copulation rate was significantly higher in the light condition $\left(X_{7}^{2}=16.99, P=0.018, R^{2}=0.214\right)$ and $\mathrm{HD}$ males were more likely to copulate than LD males in the dark condition (Pearson $X^{2}=3.895, P=0.048$ ) but not in the light (Pearson $X^{2}=0.964, P=0.326$ ). (B) Panel shows the average latency from the start of trials until copulation for HD and LD males in the $(\mathrm{V}+/ \mathrm{S}+)$ and $(\mathrm{V}-/ \mathrm{S}+)$ conditions. The latency to copulation was significantly shorter in the $(\mathrm{V}+/ \mathrm{S}+)$ condition $\left(X_{2}^{2}=8.32, P<0.016\right)$. $* P$ value of $<0.05$. Means and standard errors are presented in Panel $B$.

this mating advantage was not realized in the light $(\mathrm{V}+$ / $\mathrm{S}+$ ). These results could be the consequence of either differential plasticity in male courtship behavior for HD and LD males, or context-dependent female mate choice. To rule out the possibility that $\mathrm{HD}$ and LD males behave dif- ferently in the dark versus light (e.g., HD males are more likely to approach and mount a female in the dark as compared to LD males), we conducted a second experiment in which we temporarily visually masked males-mimicking a male's dark treatment. Mate choice trials of masked and unmasked males were then run in $\mathrm{V}+$ and $\mathrm{V}$ - conditions, always in the presence of a seismic signal (S+). If our previous results were due to differential male plasticity, we would expect to see similar mating patterns between the masked $\mathrm{V}+$ and both the masked and unmasked V- condition (HD males should have a mating advantage in the following treatments: masked $\mathrm{V}+$, masked $\mathrm{V}-$, unmasked $\mathrm{V}-$ ). If, however, our results were a result of environmentspecific female choice, we would expect to see differences in mating patterns between the light and dark treatment regardless of male masking treatment (e.g., HD males should only have a mating advantage in $\mathrm{V}$ - treatments).

\section{Experiment 3: Methods}

A total of 94 females (all LD diet treatment due to the timing of follow-up experiment) and 94 males (Masked LD $N=22$; Masked HD N = 24; Unmasked LD = 26; Unmasked HD = 22), not used previously, were each used once in mate choice trials. Trials were conducted as in the signal efficacy experiment (Experiment 2) with two modifications. First, all trials were run on filter paper, allowing seismic signal transmission (S+), and the only environmental manipulation was the presence $(\mathrm{V}+, N=45)$ or absence $(\mathrm{V}-, \mathrm{N}=49)$ of light. Second, we randomly selected half of each of our HD and LD males and temporarily masked their eyes with paint (masked condition), preventing them from receiving visual input. Eye painting has been used previously with success in numerous studies exploring spider vision (Forster 1979, 1982; Rovner 1996). To mask the eyes, males were physically restrained in a Ziplock plastic bag. A small hole was cut in the center of the bag and the male was positioned so that his eyes were exposed through this hole. All eight eyes of masked males were painted using a paintbrush dipped in black ink from a DecoColor paint marker (Uchida of America Corp, Torrance, CA). The eyes of unmasked males were painted over using a paintbrush lacking paint. Preliminary painting trials revealed that males could successfully groom off the paint within $24 \mathrm{~h}$. We noted no difference in overall grooming behavior between masked and unmasked males and all males engaged in courtship in the presence of mature female silk. The paint remained on the eyes for at least $3 \mathrm{~h}$ and thus, mate choice trials were performed $2 \mathrm{~h}$ after painting or mock painting.

\section{Experiment 3: Results}

To examine the relationship between the male masking treatment, diet treatment, and signaling environment on copulation success, we ran a nominal logistic regression, with male masking treatment, male diet, signaling 


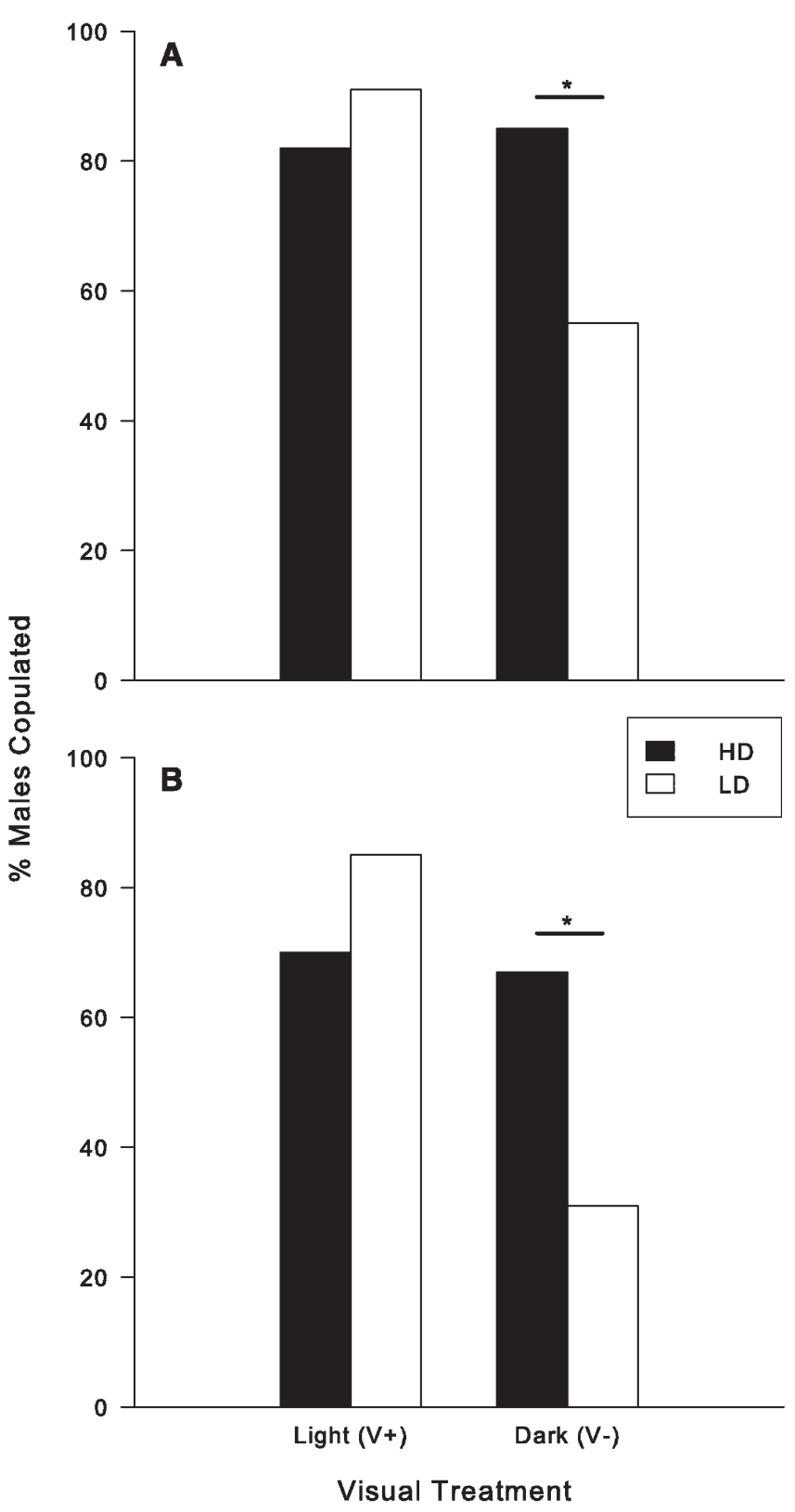

Figure 3. The proportion of HD and LD males in the $(A)$ masked and $(B)$ unmasked conditions who copulated in the light $(\mathrm{V}+/ \mathrm{S}+)$ and dark $(\mathrm{V}-/ \mathrm{S}+)$ testing conditions. Copulation rate was significantly higher in the light condition $\left(X_{1}^{2}=5.95, P<0.015\right)$ and $\mathrm{HD}$ males were more likely to copulate than LD males in the dark condition $\left(X^{2}=5.97, P=0.015\right)$ but not in the light $\left(X^{2}=0.98, P=0.322\right)$. $P P$ value of $<0.05$.

environment, and both female and male age as predictors of the occurrence of copulations. The overall model was significant $\left(X_{9}^{2}=18.45, R^{2}=0.161, P=0.03\right)$. Male diet, male or female age, and masking treatment alone did not influenced copulation frequency (Table 3B). The visual signaling environment, however, did predict copulation frequency (Figures 3A, B; Table 3B), with copulations occurring more frequently in the visual present condition (as seen in the signal efficacy experiment). We also found an interaction between the visual signaling environment and male diet, with HD males more likely to copulate in the dark than LD males, and no significant difference in likelihood to copulate between the two diet treatments in the light (Figure 3A, B; Table 3).

Age at the time of testing ranged from 15 to 27 days post maturation $(\bar{x}=22.5)$ for females and $21-35$ days $(\bar{x}=26.2)$ for males. Due to the later maturation of low-diet individuals, HD males were significantly older than LD males at the time of testing (see Table $\mathrm{S}_{1} \mathrm{~B}_{1}$ ), but as in the signal efficacy experiment there was no significant age difference in males or females who mated compared to those who did not (Table $\mathrm{S}_{1} \mathrm{~B}_{2}$ ). Furthermore, age of males and females was not significant predictors in our nominal logistic regression.

Neither male diet nor masking treatment influenced male courtship - there was no significant difference between either HD and LD males or masked and unmasked males with regard to the time from the start of the trial to their first bout of courtship, the time from the start of the trial to copulation, the time between the first bout of courtship and copulation, or the male's amount of courtship as measured by instantaneous sampling (Table S2B $\mathrm{B}_{1}$ ). Similarly, across both masking conditions, the time to the first bout of courtship did not differ significantly between the two visual treatments, however, the amount of courtship was greater in the visual present condition (Wilcoxon test: $X^{2}=2.59, P=0.009$; Table S2B ${ }_{2}$.

\section{Experiment 4: Female Fitness Effects of Mate Choice}

Due to the difference in female mate choice observed across visual signaling environments in Experiments 2 and 3, we were interested in whether these mating decisions had observable effects on offspring number. Any differences observed in offspring number could be generated by either differences in male mate quality or to differential female investment. To determine first whether a female's fitness is dependent upon her mating environment, we examined the number of offspring produced by females following their mate choice trials in Experiments 2 and 3.

\section{Experiment 4: Methods}

All copulating pairs from the previous experiments were allowed to copulate until natural cessation (range from $\sim 20 \mathrm{~min}$ to $\sim 2 \mathrm{~h}$ ). Upon completion of mate choice trials (Experiments 2 and 3), any pairs still copulating were transferred to a holding cage in a lighted room. Thus, regardless of whether a pair began copulation in the light or dark, their post trial copulation experiences were identical and copulation duration did not appear to vary with mating environment. Mated females were returned to their home cage and provisioned with two popsicle sticks to provide a more heterogeneous, enriched environment. These 
females were provided with two crickets per week, sizedmatched to the spider, and were checked daily for the production of egg sacs and the subsequent emergence of spiderlings. Upon emergence, spiderlings were removed from the mother's back, sacrificed, and counted. Because female wolf spiders can produce more than one egg sac, females were returned to their housing containers and continually monitored for additional egg sac production.

\section{Experiment 4: Results}

Of the 99 females that mated across both experiments, 83 produced at least one egg sac $(84 \%)$. Significantly more mated females produced egg sacs from the masking experiment (Experiment 3) versus the signal efficacy experiment (Experiment 2$)\left(\right.$ masking $=91 \%$, signal efficacy $=70 \% ; \chi^{2}=$ $6.886, P=0.009)$. Of the 83 females that successfully produced an egg sac, seven of the matings were artificially cut short (i.e., copulating pairs were moved and pairs were accidentally broken up during transit), whereas the remaining 76 copulations were allowed to continue until their natural cessation. The offspring number from the seven broken copulations did not differ significantly from the remaining 76 and thus, all offspring data were combined in further analyses. The total offspring number for combined data was not normally distributed and thus was square root transformed to achieve normality.

Because females appear to be choosing males differently in the light versus dark, and egg sac number varied across experiments, we used a least squares regression to exam- ine the influence of experiment (signal efficacy vs. masking) and signaling environment $(\mathrm{V}+\mathrm{vs} . \mathrm{V}-)$ on offspring number. Our overall model was significant $\left(F_{3,79}=4.4, P\right.$ $=0.006$ ). We found that although females from the masking experiment were more likely to produce an egg sac, females from the signal efficacy experiment produced more offspring (Figure 4). This main effect was driven by the increase in offspring number by females in the dark trials of the signal efficiency experiment, which was evidenced by a significant interaction between signaling environment and experiment on the total number of offspring (Figure 4). We found no influence of signaling environment alone $(F=$ $3.36, P=0.07)$.

To further explore the interaction between experiment (signal efficiency vs. masking), signaling environment ( $\mathrm{V}+$ vs. $\mathrm{V}^{-}$) and offspring number, we examined the effect of signaling environment on offspring number for each experiment separately. We found that in the signal efficacy experiment, signaling environment significantly influenced the total number of offspring a female produced $\left(F_{1,21}=5.4\right.$, $P=0.03)$, whereas the signaling environment had no influence on the total number of offspring produced in the masking experiment $\left(F_{1,58}=2.0, P=0.17\right)$. Females from the signal efficacy experiment produced more offspring when they mated in the $\mathrm{V}-$ versus $\mathrm{V}+$ condition, following our mate choice pattern of increased choosiness in the V- versus $\mathrm{V}+$ condition.

Both experiments were run under identical laboratory conditions using the same methods. Because the masking experiment was conducted as a follow-up to the signal effi-

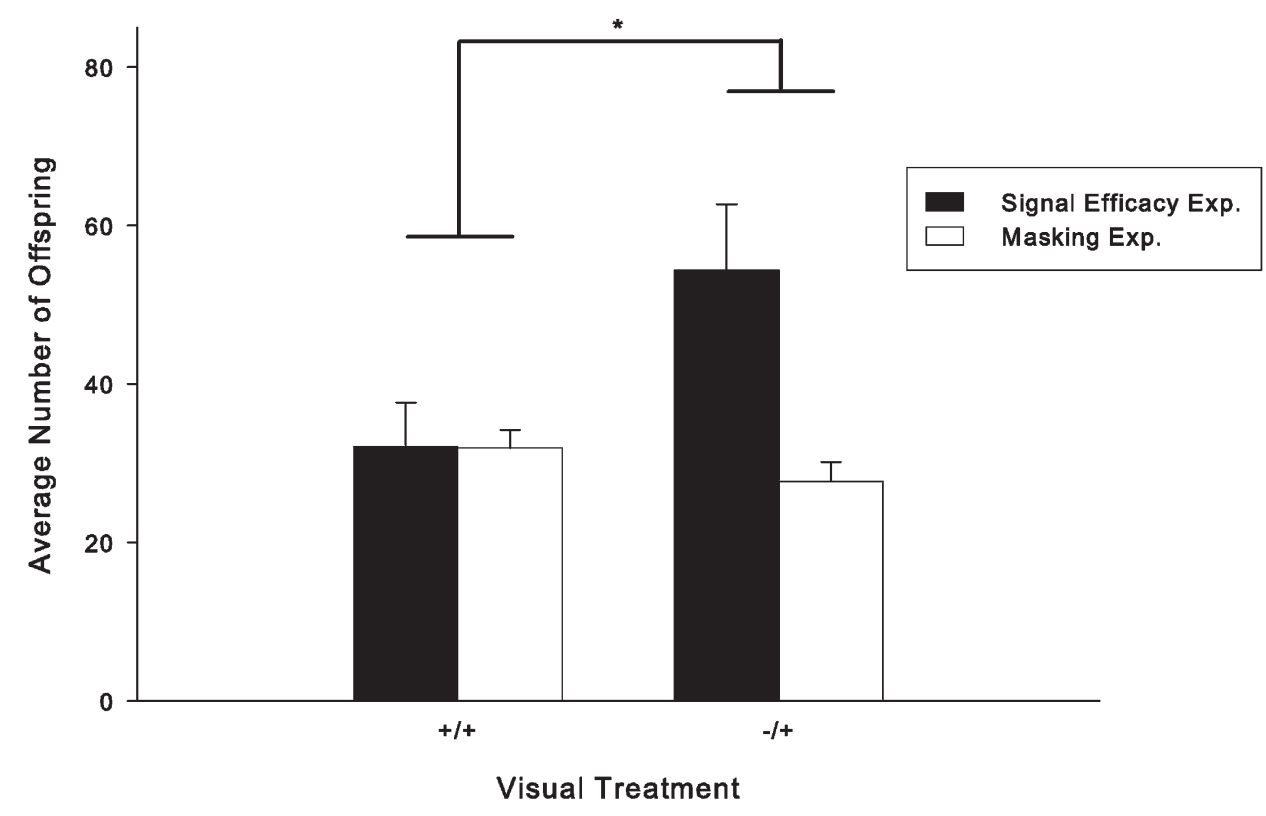

Figure 4. The average number of offspring from females in the signal efficacy and male masking experiments in the light $(\mathrm{V}+/ \mathrm{S}+)$ and dark $(\mathrm{V}-/ \mathrm{S}+)$ testing conditions. Females in the signal efficacy experiment produced significantly more offspring than in the male masking experiment $(F=5.35, P=0.02)$. Furthermore, there was a significant interaction between the testing condition and experiment $(F=10.1, P=0.002)$ with more offspring produced in the signal efficacy experiment but only in the dark condition. *P value of $<0.05$. Means and standard errors are presented. 

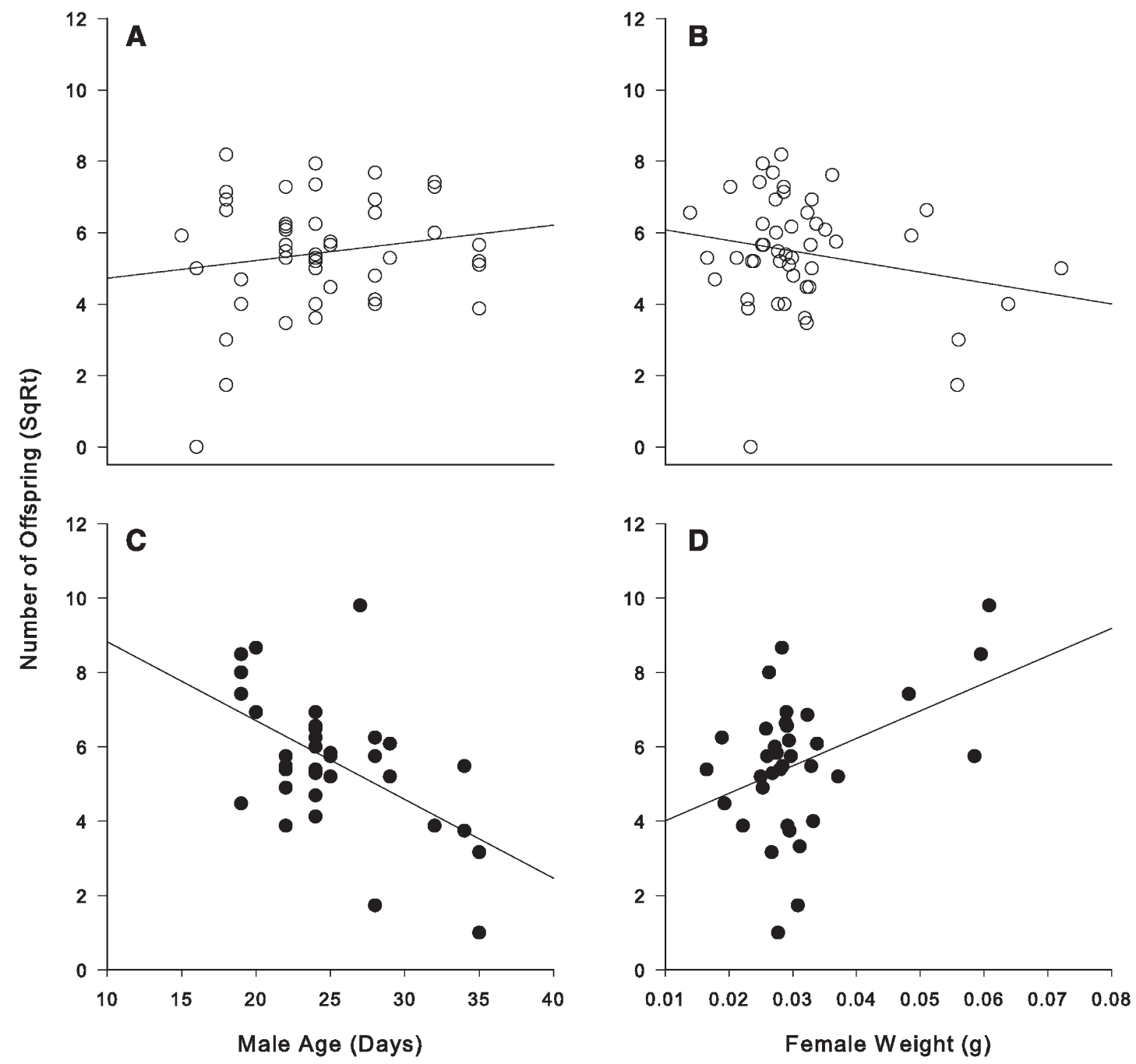

Figure 5. Least squares regression plots of the total number of offspring produced by females (square root transformed) in the light ( $V+/ \mathrm{S}+$; open circles) and dark ( $\mathrm{V}-/ \mathrm{S}+$; filled circles) testing conditions. Panels $\mathrm{A}$ and $\mathrm{C}$ show number of offspring by the age of the male they mated with and Panels $B$ and $D$ show number of offspring by the weight of the female. The number of offspring decreases with male age in the dark but increases in the light. Furthermore, in the dark the number of offspring increases with female weight with the opposite trend in the light.

cacy experiment, it was necessarily run later in the season. Thus, the differences between the two experiments were the following. (1) Females used in the masking experiment were approximately 2 days older than those used in the signal efficacy experiment (Wilcoxon test $\chi^{2}=4.1, P=0.04$; Table S1). (2) Males were approximately 6 days older in the masking experiment versus the signal efficacy experiment (Wilcoxon Test $\chi^{2}=21.42, P<0.0001$; Table S1). Additionally, (3) in the masking experiment only LD females were used, generating a significant difference in female weight between the two experiments (signal efficacy: $\bar{x}=0.039 \pm$ $0.002 \mathrm{~g}$, masking: $\bar{x}=0.028 \pm 0.001 \mathrm{~g}$; $t$-test, $t=4.366, \mathrm{df}$ $=81, P<0.0001)$. Given that female age did not influence mating in either experiment, and given that the difference in age was only 2 days (making it unlikely that this difference is biologically meaningful here), we sought to further pursue the influence of male age and female weight on female fitness.
To simultaneously explore the effects of male age, female weight, and signaling environment on the total number of offspring produced, we ran a least squares regression model with the following three parameters and all possible interactions: signaling environment, female weight, and male age. We found that this model including the three-way interaction between signaling environment, female weight, and male age explained our results $\left(R^{2}=\right.$ $\left.0.25, F_{7,82}=3.79, P=0.002\right)$. When we explored offspring production for each signaling environment separately, we found no influence of either male age or female weight in the light (whole model, $F_{3,44}=0.81, P=0.5$ ), but we found an influence of male age and female weight on offspring production in the dark, with no interaction between the two (whole model $F_{3,31}=8.8, P=0.0002$; male age, $F=14.6$, $P=0.0006$; female weight, $F=9.8, P=0.004$; male age $\times$ female weight, $F=1.0, P=0.3$; Figure 5). Importantly, we found no relationship between the number of offspring 
produced by a female and the body condition score of the male (Bivariate analysis: $F=2.13, \mathrm{df}=79, P=0.146$ ).

\section{Discussion}

Male S. floridana wolf spiders incorporate both visual and seismic components into their courtship display. Here, using a combination of approaches, we first demonstrate that components of both signaling modalities encode information about a male's body condition, making them at least partially redundant in terms of encoded information. Our males raised on high-quantity diets (HD) had both darker foreleg segments and a higher percentage of their foreleg segments covered with dark coloration than those males raised on low-quantity diets (LD). Similarly, we found significant differences in seismic courtship components between HD and LD males - for example, HD males produced more pulses per minute and repeated them at a higher rate within a signaling bout. Our mating trials conducted across signaling environments, however, demonstrate that these signals are nonredundant in terms of receiver response. The seismic signal for $S$. floridana appears crucial for mating success, a result that has been found consistently across several other Schizocosa species (Scheffer et al. 1996; Hebets and Uetz 1999; Hebets 2005, 2008; but see Rundus et al. 2010). In contrast to the importance of the seismic signal, the visual courtship components appeared neither necessary nor sufficient for successful mating. Most interestingly, however, we found environment-dependent mating success for males of varying body condition. In the dark, HD males achieved more copulations than LD males, but they achieved equivalent copulation frequencies in the light. A follow-up experiment in which we reversibly visually masked males and ran mating trials across signaling environments supported the notion that females indeed are choosier in the dark - presumably basing their mating decisions solely on a male's seismic courtship signal. Finally, we found that these mating decisions have fitness consequences. In the dark mating environments, where females were choosier, female weight correlated with offspring number, whereas no such correlation was observed with females mating in the light condition. Taken together, our results demonstrate context or environment-dependent female reliance on courtship components and suggest that this plasticity in female mate choice has direct female fitness consequences.

Condition-dependent ornament expression has been documented in other Schizocosa species (Uetz et al. 2002; Hebets et al. 2008; Shamble et al. 2009), but to date, condition-dependent seismic signal production has been suggested in only S. ocreata (Gibson and Uetz 2008). Despite the condition-dependent nature of ornament expression across other Schizocosa species, however, females of these ornamented species do not always choose males based upon these visual secondary sexual traits (McClintock and
Uetz 1996; Scheffer et al. 1996; Shamble et al. 2009, but see Persons and Uetz 2005). Similarly, in S. floridana, male foreleg pigmentation can potentially inform females of a male's body condition. Although other visual components of male courtship were not tested for condition dependence, females do not appear to base mate choice decision on visual aspects of courtship, as females did not choose HD males over LD males in environments in which they could assess this visual signal $(\mathrm{V}+$ signaling environments, see Figure 2). Furthermore, additional analysis of our results revealed that male foreleg pigmentation in no way correlates with male mating success (see Supporting information). Nonetheless, when seismic signals were present $(\mathrm{S}+)$, females mated more frequently and more quickly in the presence versus absence of the visual signal. Unfortunately, our data do not allow us to determine whether the visual courtship components per se played a role in these resulting differences. One obvious equally parsimonious explanation exists - a female's perceived predation risk may be higher in the light versus dark and thus, her motivation to mate may be higher under these conditions, leading to a potential trade-off between time for mate assessment and time exposed to predation risk. In the light, females may forego mate assessment in lieu of reduced exposure time to potential predators.

Although we did not detect female choice based upon male foreleg pigmentation, we did detect female choice based upon male seismic signals - but only in the absence of visual signals. This result is intriguing as it suggests that females attend to different signal components across different signaling environments-making mate choice decisions based upon seismic signals only in the dark. This suggestion of environment-dependent mate choice is dependent upon the fact that our observed mating patterns are the result of female choice and not environment-specific differences in male courtship behavior. To rule out this latter possibility, we conducted a subsequent experiment in which males were either reversibly blinded or not and were run through mating trials in either the presence or absence of the visual signal (V+ vs. $V-$, both with seismic signal present). We predicted that if HD males were more persistent in obtaining copulations than LD males in the dark, we would see similar mating frequencies in the masked $\mathrm{V}+$ males and both the masked and unmasked V- males. However, we found no effect of masking and instead, we again found that HD males achieved more copulations than LD males only in the visual absent signaling environments. Although female-male interactions were undoubtedly influenced by male masking, our results support the hypothesis that our observed pattern of mating frequencies result from female, not male-driven differences.

Context, or environment-dependent female mating preferences are not uncommon, as females of various taxa exhibit a reduction in choosiness when exposed to a predator (e.g., Forsgren 1992; Godin and Briggs 1996; Gong and Gibson 1996; Johnson and Basolo 2003) or simply with per- 
ceived predation risk. For example, in crickets, females are less likely to choose a previously preferred call when it is associated with little or no cover (Hedrick and Dill 1993). The same, or a similar, explanation may be applied to our findings. As suggested previously, in the light, females may perceive their risk of predation to be higher and thus, may opt to forego male assessment and mate more quickly. Ultimately, the same seismic information is available to females in the light, but females may ignore this information in exchange for a quicker mating. An alternative explanation is that females attend to different signal components or combinations of components across environmental conditions. For example, when only one signal can be perceived, females might attend solely to that single signal, yet when multiple signals can be received simultaneously, females may alter their attention to different signals or signal combinations. Female $S$. uetzi have been shown to alter their reliance on visual signals only in the presence of seismic signals (Hebets 2005) and female sticklebacks switch between relying on visual versus olfactory cues depending upon environmental conditions (Heuschele et al. 2009). However, given that the visual and seismic signals appear to convey similar information in S. floridana, and that HD males did not achieve more matings in the light, this latter explanation seems less likely.

Regardless of the mechanism underlying the different mating strategies of $S$. floridana females across signaling environments, results from two separate experiments here suggest that females mating in the dark are choosier than those mating in the light. The next step then was to determine the fitness consequences of these different mating decisions. Data from our first experiment (signal efficacy) demonstrated that females that mated in the dark treatment produced significantly more offspring than those mating in the light. However, in our follow-up experiment (male masking), we did not observe mating environment-dependent offspring number. The main differences between these experiments involved male age and female weight (males were older and females were lighter in the follow-up masking experiment). These differences were unavoidable as this second experiment was unplanned and the only available naïve virgin individuals at the time were older males and LD females. Not surprisingly then, when we combined our datasets and looked for variables predictive of offspring number, we found that both male age and female weight influenced offspring number, but only in the dark mating trials. The pattern indicated that older males were associated with less offspring in the dark whereas heavier females produced more offspring in the dark, with no interaction between the two. The fact that male age and female weight correlate with offspring number only in the dark mating trials, the trials in which females were choosier, suggests that females, not males, are responsible for these differences. The data presented here raise the distinct possibility that females can vary their reproductive investment based upon the perceived quality of their mates and that this variance in reproductive investment is condition-dependent. Female spiders have two phases of egg yolking, the second of which starts immediately after copulation (Foelix 1996). If females are varying their reproductive investment (e.g., more eggs yolked up; more yolk per egg; etc.) then we might expect to see females with sufficient resources (HD females) investing more when conditions allow for increased choosiness $\left(\mathrm{V}^{-}\right)$compared to $(\mathrm{V}+)$ conditions in which these high-quality females may choose to allocate extra resources to a potential future mating. Although the majority of female Schizocosa wolf spiders mate only once in their lifetime, previous studies have found that a small proportion of females, $14 \%$ or less, mate a second time (Norton and Uetz 2005; Persons and Uetz 2005).

In a recent study using another wolf spider (Pardosa milvina), Hoefler et al. (2009) found that females that mated with males courting at a high rate had more offspring that emerged sooner and survived better than offspring from females that mated with males of a low courtship rate. They suggested that their results provide evidence of indirect benefits to female via mating with males with high courtship rates, yet they allow for the possibility that females might be manipulating these fitness measures. Another recent study on spitting spiders (family Scytodidae) also found that females mating with preferred males produced more eggs that were larger in size and had greater fertility (Koh et al. 2009). These authors also suggest that females gain indirect fitness benefits by mating with preferred males, without mention of differential female reproductive investment as an alternative explanation. Based upon the results of our study, we suggest that such findings deserve further exploration to determine whether fitness differences are female or male driven. Our results suggest that it is indeed the females, and not the males, that are responsible for the differences in offspring number across mating environments, as offspring number did not vary with male body condition.

In summary, we provide a comprehensive set of experiments exploring the signal content, signal efficacy, and fitness consequences of mating decisions in the multimodal signaling wolf spider $S$. floridana. We demonstrate condition-dependent foreleg ornamentation and seismic signaling; we further demonstrate that female mating decisions are environment-dependent; and we finally demonstrate that female mating decisions directly influence female fitness. Our findings suggest that females may differentially attend to signal components across environments, suggesting that selection acting on particular signal components may only be realized in specific signaling environments. This environment-dependent selection, in addition to variability in female choosiness both within and across environments, may help explain the abundance of additive genetic variation in male secondary sexual traits.

Finally, our results are among the first to demonstrate fitness consequences of mating decisions based upon select signal components from complex displays. Although 
further research is clearly necessary regarding the fitness consequences of mating in S. floridana, we suggest that future studies exploring complex signal function should attempt to attain fitness data. Ultimately, our understanding of complex signal function and evolution will be greatly strengthened by studies that combine content-based and efficacy-based approaches while simultaneously examining receiver variability and assessing fitness outcomes.

Acknowledgments - We would like to thank G. B. Edwards for his assistance and advice on collecting $S$. floridana for this study and for K. Swoboda for the care and maintenance of spiders in the laboratory. This work was supported by the National Science Foundation [IOS - 0643179 to EAH].

\section{Literature Cited}

Ayyagari, L. R., and W. J. Tietjen. 1986. Preliminary isolation of male-inhibitory pheromone of the spider Schizocosa ocreata (Araneae, Lycosidae). J. Chem. Ecol. 13:237-244.

Barth, F. G. 2002. A spider's world: Senses and behavior. Springer, Berlin, Germany.

Candolin, U. 2003. The use of multiple cues in mate choice. Biol. Rev. 78:575- 595.

Coleman, S. W., G. L. Patricelli, and G. Borgia. 2004. Variable female preferences drive complex male displays. Nature 428:742-745.

Collins, S. A. 1995. The effect of recent experience on female choice in zebra finches. Anim. Behav. 49:479-486.

Cotton, S., K. Fowler, and A. Pomiankowski. 2004. Do sexual ornaments demonstrate heightened condition-dependent expression as predicted by the handicap hypothesis? Proc. R Soc. Lond., B 271:771-783.

DeVoe, R. D. 1972. Dual sensitivities of cells in wolf spider eyes at ultraviolet and visible wavelengths of light. J. Gen. Physiol. 59:247-269.

DeVoe, R. D., R. J. W. Small, and J. E. Zvargulis. 1969. Spectral sensitivities of wolf spider eyes. J. Gen. Physiol. 54:1-32.

Dondale, C. D., and J. H. Redner. 1978. Revision of the nearctic wolf spider genus Schizocosa (Araneida: Lycosidae). Can. Ent. 110:143-181.

Elias, D., A. Mason, and R. Hoy. 2004. The effect of substrate on the efficacy of seismic courtship signal transmission in the jumping spider Habronattus dossenus (Araneae: Salticidae). J. Exp. Biol. 207:4105-4110.

Endler, J. A. 1993. Some general comments on the evolution and design of animal communication systems. Philos. Trans. R. Soc. Lond.B340:215- 225.

Eraly, D., F. Hendrickx, and L. Lens. 2009. Condition-dependent mate choice and its implications for population differentiation in the wolf spider Pirata piraticus. Behav. Ecol. 20:856-863.

Fleishman, L. J. 1986. Motion detection in the presence and absence of background motion in an Anolis lizard. J. Comp. Physiol. A 159:711- 720.

Foelix, R. 1996. Biology of spiders, 2nd edition. Oxford Univ. Press, New York.
Forsgren, E. 1992. Predation risk affects mate choice in a gobiid fish. Am Nat. 140:1041-1049.

Forster, L. M. 1979. Visual mechanisms of hunting behaviour in Trite planiceps - a jumping spider (Araneae: Salticidae). N Z. J. Zool. 6:79- 93.

Forster, L. M. 1982. Non-visual prey-capture in Trite planiceps, a jumping spider (Araneae, Salticidae). J. Arach. 10:179-183.

Gaskett, A. C. 2007. Spider sex pheromones: Emission, reception, structures, and functions. Biol. Rev. 82:27-48.

Gibson, J., and G. Uetz. 2008. Seismic communication and mate choice in wolf spiders: Components of male seismic signals and mating success. Anim. Behav. 75:1253-1262.

Godin, J. G. J., and S. E. Briggs. 1996. Female mate choice under predation risk in the guppy. Anim. Behav. 51:117-130.

Gong, A., and R. M. Gibson. 1996. Reversal of female preference after visual exposure to a predator in the guppy, Poecilia reticulata. Anim. Behav. 52:1007-1015.

Guilford, T., and M. Dawkins. 1991. Receiver psychology and the evolution of animal signals. Anim. Behav. 42:1-14.

Hebets, E. A. 2003. Subadult experience influences adult mate choice in an arthropod: Exposed female wolf spiders prefer males of a familiar phenotype. Proc. Natl. Acad. Sci. USA 100:13390-13395.

Hebets, E. A. 2005. Attention-altering signal interactions in the multimodal courtship display of the wolf spider Schizocosa uetzi. Behav. Ecol. 16:75-82.

Hebets, E. A. 2008. Seismic signal dominance in the multimodal courtship display of the wolf spider Schizocosa stridulans Stratton 1991. Behav. Ecol. 19:1250-1257.

Hebets, E. A., and D. Papaj. 2005. Complex signal function: Developing a framework of testable hypotheses. Behav. Ecol. Sociol. 57:197-214.

Hebets, E. A., and G. Uetz. 1999. Female responses to isolated signals from multimodal male courtship displays in the wolf spider genus Schizocosa (Araneae: Lycosidae). Anim. Behav. 57:865-872.

Hebets, E. A., and C. J. Vink. 2007. Experience leads to preference: Experienced females prefer brush-legged males in a population of syntopic wolf spiders. Behav. Ecol. 18:1010-1020.

Hebets, E. A., J. Wesson, and P. S. Shamble. 2008. Diet influences mate choice selectivity in adult female wolf spiders. Anim. Behav. 76:355- 363.

Hedrick, A. V., and L. M. Dill. 1993. Mate choice by female crickets is influenced by predation risk. Anim. Behav. 46:193-196.

Heuschele, J., M. Mannerla, P. Gienapp, and U. Candolin. 2009. Environment-dependent use of mate choice cues in sticklebacks. Behav. Ecol. 20:1223-1227.

Hoefler, C. D., A. L. Carlascio, M. H. Persons, and A. L. Rypstra. 2009. Male courtship repeatability and potential indirect genetic benefits in a wolf spider. Anim. Behav. 78:183-188.

Jennions, M. D., and M. Petrie. 1997. Variation in mate choice and mating preferences: A review of causes and consequences. Biol. Rev. 72:283- 327.

Johnson, J. B., and A. L. Basolo. 2003. Predator exposure alters female mate choice in the green swordtail. Behav. Ecol. 14:619-625. 
Johnstone, R. A. 1996. Multiple displays in animal communication: 'backup signals' and 'multiple messages'. Philos. Trans. R. Soc. Lond. B 351:329-338.

Koh, T. H., W. K. Seah, L. Yap, and D. Q. Li. 2009. Pheromone-based female mate choice and its effect on reproductive investment in a spitting spider. Behav. Ecol. Sociol. 63:923-930.

Møller,A. P., and A. Pomiankowski. 1993. Why have birds got multiple sexual ornaments. Behav. Ecol. Sociol. 32:167-176.

Moore, P. J., and A. J. Moore. 2001. Reproductive aging and mating: The ticking of the biological clock in female cockroaches. Proc. Natl. Acad. Sci. USA 98:9171-9176.

McClintockW. J., and G.W. Uetz. 1996. Female choice and preexisting bias: Visual cues during courtship in two Schizocosa wolf spiders (Araneae: Lycosidae). Anim. Behav. 52:167-181.

Norton, S., and G. W. Uetz. 2005. Mating frequency in Schizocosa ocreata (Hentz) wolf spiders: Evidence for a mating system with female monandry and male polygyny. J. Arach. 33:16-24.

Owings, D. H., and E. S. Morton. 1998. Animal vocal communication: A new approach. Cambridge Univ. Press, Cambridge, New York.

Partan, S., and P. Marler. 1999. Communication goes multimodal. Science 283:1272-1273.

Partan, S., and P. Marler. 2005. Issues in the classification of multimodal communication signals. Am Nat. 166:231-245.

Persons, M. H., and G. W. Uetz. 2005. Sexual cannibalism and mate choice decisions in wolf spiders: Influence of male size and secondary sexual characters. Anim. Behav. 69:83-94.

Rovner, J. S. 1996. Conspecific interactions in the lycosid spider Rabidosa rabida: The roles of different senses. J. Arach. 24:16-23.

Rundus, A. S., R. D. Santer, and E. A. Hebets. 2010. Multimodal courtship efficacy of Schizocosa retrorsa wolf spiders: Implications for near-field communication. Behav Ecol. 21:701-707.

Scheffer, S., G. Uetz, and G. Stratton. 1996. Sexual selection, male morphology, and the efficacy of courtship signalling in two wolf spiders (Araneae: Lycosidae). Behav. Ecol. Sociol. 38:17-23.

Shamble, P. S., D. J. Wilgers, K. A. Swoboda, and E. A. Hebets. 2009. Courtship effort is a better predictor of mating success than ornamentation for male wolf spiders. Behav. Ecol. 20:1242-1251.

Stratton, G. 2005. Evolution of ornamentation and courtship behavior in Schizocosa: Insights from a phylogeny based on morphology (Araneae, Lycosidae). J. Arach. 33:347-376.

Tobler, M., I. Schlupp, and M. Plath. 2008. Does divergence in female mate choice affect male size distributions in two cave fish populations? Biol. Lett. 4:452-454.

Uetz, G., and S.Norton. 2007. Preference formale traits in female wolf spiders varies with the choice of available males, female age and reproductive state. Behav. Ecol. Sociol. 61:631-641.

Uetz, G. W., R. Papke, and B. Kilinc. 2002. Influence of feeding regime on body size, body condition and a male second- ary sexual character in Schizocosa ocreata wolf spiders (Araneae, Lycosidae): Condition-dependence in a visual signaling trait. J. Arach. 30:461- 469.

Wagner, W. E., M. R. Smeds, and D. D. Wiegmann. 2001. Experience affects female responses to male song in the variable field cricket Gryllus lineaticeps (Orthoptera, Gryllidae). Ethology 107:769-776.

\section{Supplementary Material: Effects of Ornamentation on Copulation Success}

\section{Methods}

In order to determine whether foreleg ornamentation influences copulation success, we examined the relationship between foreleg ornamentation and copulation success for males run in the following signaling environment - visual present/seismic present $(\mathrm{V}+/ \mathrm{S}+)$. Since all males from the masking experiment (Experiment 2) were preserved identically (i.e. they were frozen at $-4^{0} \mathrm{C}$ ) we chose to examine only males from this experiment. Briefly, using the protocol described previously (Experiment 1 - Quantifying foreleg ornamentation - METHODS), we quantified the foreleg ornamentation for all males used in the V+ treatment of Experiment 2 .

\section{Results}

The ornamented segments of male forelegs in the visual present condition were significantly different between the diet treatments as indicated by the average darkness across both the tibia and metatarsus (average ornamentation darkness; HD: $N=21, \bar{x}=59.0 \pm 1.3$; LD: $N=21, \bar{x}=$ $\left.65.5 \pm 1.3 ; t_{40}=3.61, P<0.001\right)$. However, despite these differences, and despite the fact that foreleg pigmentation is condition-dependent (see RESULTS Exp. 1), the average ornamentation darkness had no influence on overall copulation success (successful males: $N=34, \bar{x}=62.1 \pm 2.4$; unsuccessful males: $N=8, \bar{x}=62.6 \pm 1.2 ; t_{40}=0.2, P=0.84$ ) or latency to copulation from courtship initiation $\left(N=34, r^{2}=\right.$ $0.08, P=0.12)$. Even within diet treatments, males successful at gaining copulations were no darker than unsuccessful (HD: successful: $N=16, \bar{x}=58.8 \pm 1.2$, unsuccessful: $N$ $=5, \bar{x}=59.6 \pm 2.2 ; t_{19}=0.32, P=0.75 ;$ LD: successful: $N=$ $18, \bar{x}=65.1 \pm 1.6$, unsuccessful: $N=3, \bar{x}=67.7 \pm 3.9 ; t_{19}=$ $0.63, P=0.54)$. Since males used in this experiment were either masked or not, we also ran a nominal logistic regression for masking treatment, average ornamentation, and an interaction between masking treatment and ornamentation on likelihood to copulate and our model was not significant $\left(\mathrm{X}^{2}=1.2, \mathrm{df}=3, p=0.75\right)$. 


\section{Supplementary Tables}

Table SI. The average age (days post maturation molt) of males and females. Means \pm SE are shown.

\section{(A) Signal Efficacy Experiment.}

$\mathbf{A}_{1}$ : High diet and low diet treatments and the combined age irrespective of diet treatment.

\begin{tabular}{|c|c|c|c|c|c|}
\hline & Combined & High diet & Low diet & Wilcoxon Z & $P$ value \\
\hline Female & $20.6 \pm 0.68$ & $22.82 \pm 0.42$ & $19.06 \pm 0.46$ & 5.76 & $<0.001$ \\
\hline Male & $20.5 \pm 0.89$ & $22.04 \pm 0.57$ & $18.76 \pm 0.44$ & 4.47 & $<0.001$ \\
\hline \multicolumn{6}{|c|}{$\mathbf{A}_{2}$ : Trials in which and mating did or did not occur. } \\
\hline & Trials with mating & & ut mating & Wilcoxon Z & $P$ value \\
\hline Male & $20.53 \pm 0.80$ & & 0.44 & 0.21 & 0.834 \\
\hline
\end{tabular}

\section{(B) Masking Experiment.}

$\mathbf{B}_{\mathbf{I}}$ : High diet and low diet treatments and the combined age irrespective of diet treatment.

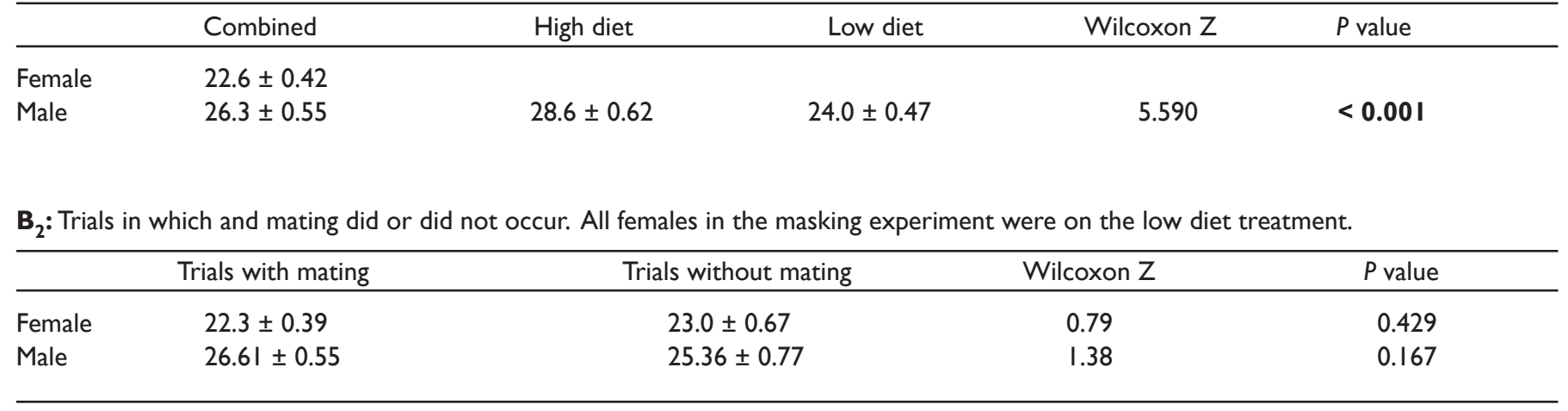


Table S2. Courtship effort by groups of males, measured as the time to the first courtship bout (s), the time to copulation (s), the rate of courtship (\# instantaneous 5 minute samples in which courtship occurred), and the time from the first courtship bout to copulation (s). Means \pm SE are shown. Kruskal-Wallis $\chi^{2}$ tests were used in the signal efficacy experiment while Wilcoxon $Z$ tests were used in the masking experiment, as there were only two environmental treatment conditions in the latter.

\section{(A) Signal Efficacy Experiment.}

$\mathbf{A}_{1}$ : Differences among high diet and low diet treatments

\begin{tabular}{|c|c|c|c|c|c|c|}
\hline & \multicolumn{2}{|c|}{ High diet } & Low diet & \multicolumn{2}{|l|}{ Wilcoxon Z } & $P$ value \\
\hline Time to copulate & \multicolumn{2}{|c|}{$547.8 \pm 54.6$} & $473.0 \pm 66.1$ & 1.66 & \multicolumn{2}{|r|}{0.096} \\
\hline Amount of courtship & \multicolumn{2}{|c|}{$1.9 \pm 2.4$} & $2.0 \pm 0.32$ & 0.46 & \multicolumn{2}{|r|}{0.647} \\
\hline \multicolumn{7}{|c|}{$\mathbf{A}_{\mathbf{2}}$ : Differences across environmental treatments. } \\
\hline Time to courtship & $97.7 \pm 40.6$ & $216.5 \pm 41.4$ & $60.4 \pm 37.9$ & $44.9 \pm 43.1$ & 22.2 & $<0.0001$ \\
\hline Amount of courtship & $0.71 \pm 0.08$ & $0.56 \pm 0.07$ & $0.64 \pm 0.06$ & $0.76 \pm 0.05$ & 4.01 & 0.261 \\
\hline
\end{tabular}

(B) Masking Experiment.

B $_{\text {I }}$ Differences among high diet and low diet treatments

\begin{tabular}{lcccccccc}
\hline & High diet & Low diet & $Z$ & $P$ value & Masked & Unmasked & Wilcoxon Z & $P$ value \\
\hline Time to courtship & $78.7 \pm 19.0$ & $129.0 \pm 37.9$ & 1.19 & 0.235 & $36.11 \pm 6.69$ & $49.79 \pm 9.93$ & 1.14 & 0.255 \\
Time to copulate & $611.9 \pm 122.2$ & $468.4 \pm 72.1$ & 0.54 & 0.588 & $522.4 \pm 57.3$ & $501.0 \pm 64.0$ & 0.32 & 0.747 \\
Courtship to copulation & $561.4 \pm 115.4$ & $375.6 \pm 72.5$ & 0.86 & 0.391 & $483.61 \pm 57.2$ & $449.07 \pm 65.6$ & 0.7 & 0.483 \\
Amount of courtship & $2.86 \pm 0.29$ & $2.64 \pm 0.28$ & 0.54 & 0.590 & $1.8 \pm 0.27$ & $2.2 \pm 0.29$ & 0.81 & 0.417
\end{tabular}

$\mathbf{B}_{2}$ : Differences across environmental treatments.

\begin{tabular}{lcccc}
\hline & $V+/ S+$ & $V-/ S+$ & Chi square & $P$ value \\
\hline Time to courtship & $52.9 \pm 11.4$ & $34.02 \pm 4.78$ & 0.82 & 0.415 \\
Amount of courtship & $1.5 \pm 0.28$ & $2.5 \pm 0.27$ & 2.59 & 0.009 \\
\hline
\end{tabular}

